\title{
ДИФФЕРЕНЦИРОВАННЫЙ ПОДХОД К ГЛИКЕМИЧЕСКОМУ КОНТРОЛЮ У БОЛЬНЫХ САХАРНЫМ ДИАБЕТОМ 2 ТИПА ВО ВРЕМЯ СТАЦИОНАРНОГО ЛЕЧЕНИЯ ПО ПОВОДУ ОСТРОГО ИНФАРКТА МИОКАРДА
}

\author{
Коротина М.А., Починка И.Г., Стронгин Л.Г., Ботова С.Н.
}

\author{
ФГБОУ ВО «Приволжский исследовательский медицинский университет» Минздрава России, Нижний Новгород
}

ЦЕЛЬ: оценить эффективность дифференцированного подхода к гликемическому контролю у больных сахарным диабетом 2 типа (СД2) во время стационарного лечения по поводу острого инфаркта миокарда (ОИМ).

МАТЕРИАЛЫ И МЕТОДЫ: в одноцентровое когортное исследование включены 237 больных СД2, последовательно госпитализированных по поводу ОИМ в Региональный сосудистый центр в течение 200 дней. Отдаленный исход оценивался на 365 день от момента госпитализации. На основании анализа связи между риском наступления смерти и гликемическим контролем сформулированы критерии фенотипов, требующих различных подходов к управлению гликемией во время стационарного лечения по поводу ОИМ. Эффективность дифференцированного подхода к управлению гликемией оценивалась в проспективном исследовании. Группу вмешательства составили 63 пациента СД2, госпитализированные с ОИМ, подвергнутые эндоваскулярному восстановлению кровотока в инфаркт-связанной артерии. Вмешательство заключалось в отнесении пациента к одному из 3 фенотипов: при 1 фенотипе проводилась монотерапия инсулинами, при 2 - интенсификация лечения таблетированными сахароснижающими препаратами (ТСП), при 3 фенотипе применялись ТСП в дозах, используемых пациентом до госпитализации. Критерием эффективности гликемического контроля считали значение доли измерений гликемии в пределах целевого диапазона (6,1-10,0 ммоль/л). Медиана количества измерений гликемии в течение госпитализации составила 23 [15; 33] раза.

PЕзУЛЬтАТЫ: за 12 мес наблюдения в исходной когорте зарегистрировано 53 случая смерти. Больные, у которых более 55\% измерений глюкозы во время стационарного лечения по поводу ОИМ оказывались в целевом диапазоне гликемии, риск смертельного исхода в течение года достоверно снижался: ОР 0,38 (0,18-0,77), p=0,007 (Cox regression). Предикторами неблагополучного гликемического контроля в стационаре являлись инсулинотерапия до ОИМ и гликемия при поступлении $>12,1$ ммоль/л (дискриминантный анализ). Фенотип 1 определялся по наличию 1 из критериев: гликемия при поступлении $>12,1$ ммоль/л, инсулинотерапия на догоспитальном этапе, снижение уровня сознания и острая сердечная недостаточность, скорость клубочковой фильтрации<30 мл/мин. Распределение на 2 и 3 фенотипы основывалось на соответствии $\mathrm{HbA}_{1 c}$ целевым значениям и наличию нерациональной комбинации ТСП. В группе вмешательства распределение по фенотипам было следующим: $1-41$ пациент (64\%), $2-$ 9 (14\%), 3 - 14 (22\%). Средняя гликемия в группе вмешательства и в исходной когорте достоверно не отличалась: 9,4 $\pm 2,9$ ммоль/л vs 10,0 $\pm 3,5$ ммоль/л $(p=0,2)$. При этом доля измерений в целевом диапазоне в группе вмешательства составила $63,6[53,3 ; 73,0] \%$ vs $50,0[33,3 ; 68,0] \%$ в исходной когорте (p $<0,001$, Mann-Whitney). В группе вмешательства более 55\% измерений гликемии в целевом диапазоне наблюдалось в 69\% случаев (42 пациента) vs 39\% (92 пациента) в исходной когорте, $p<0,001, \chi^{2}$ Pearson.

ВЫВоды: у больных СД2, госпитализированных с ОИМ, наличие более 55\% измерений уровня глюкозы в пределах 6,1-10,0 ммоль/л сопровождается снижением риска смерти в течение 12 месяцев. Дифференцированный подход к управлению гликемией, основанный на определении фенотипа больного СД2, приводит к улучшению гликемического контроля во время стационарного лечения по поводу ОИм.

КЛЮЧЕВЫЕ СЛОВА: гликемический контроль; сахарный диабет; острый инфаркт миокарда; фенотип. 